\title{
QUADRATIC LEVEL QUASIGROUP EQUATIONS WITH FOUR VARIABLES I
}

\begin{abstract}
Aleksandar Krapež
Abstract. We consider a class of functional equations with one operational symbol which is assumed to be a quasigroup. Equations are quadratic, level and have four variables each. Therefore, they are of the form $x_{1} x_{2} \cdot x_{3} x_{4}=$ $x_{5} x_{6} \cdot x_{7} x_{8}$ with $x_{i} \in\{x, y, u, v\}(1 \leqslant i \leqslant 8)$ with each of the variables occurring exactly twice in the equation. There are 105 such equations. They separate into 19 equivalence classes defining 19 quasigroup varieties.

The paper (partially) generalizes the results of some recent papers of Förg-Rob and Krapež, and Polonijo.
\end{abstract}

\section{Quasigroups}

One way to define a quasigroup is that it is an algebra $(S ; \cdot, \backslash, /)$ with three binary operations - multiplication $(\cdot)$, left $(\backslash)$ and right $(/)$ division, satisfying the axioms:

$$
\begin{array}{ll}
x \backslash x y=y & x(x \backslash y)=y \\
x y / y=x & (x / y) y=x .
\end{array}
$$

Very often we say that the operation · is a quasigroup assuming the underlying base set $S$ and the division operations. As usual, whenever unambiguous, the terms like $x \cdot y$ and $f(x)$ are shortened to $x y$ and $f x$ respectively.

We review a few basic facts on quasigroups. More can be found in standard references: Belousov [2], Pflugfelder [20], Chein, Pflugfelder and Smith [6].

A loop is a quasigroup with unit $(e)$, which is a value of constant terms $(x \backslash x$ and $y / y$ ) from the additional axiom:

$$
x \backslash x=y / y .
$$

The element $e$ of a loop behaves as a multiplication unit, namely

$$
e x=x e=x .
$$

2000 Mathematics Subject Classification: Primary 39B52; Secondary 20 N05.

Key words and phrases: Functional equation, quadratic equation, level equation, Belousov equation, balanced equation, gemini equation, medial equation, general solution, quasigroup, isotopy, quasigroup (left, right) linear over a group, $T$-quasigroup, variety.

Supported by the Ministry of Sciences of R. Serbia, grants 144013 and 144018. 
Groups are associative quasigroups, i.e., they satisfy:

$$
x \cdot y z=x y \cdot z
$$

and they necessarily contain a unit. A quasigroup is commutative if

$$
x y=y x
$$

and unipotent if

$$
x x=y y .
$$

Commutative groups are known as Abelian groups while unipotent groups are Boolean groups (also groups of exponent 2). Boolean groups are necessarily commutative.

A pointed quasigroup, i.e., a quasigroup with a distinguished element $e$, is skew symmetric if

$$
x y \cdot y x=e .
$$

It is a b0-quasigroup if it satisfies

$$
e x=x e
$$

and a b1-quasigroup if it satisfies

$$
e \cdot x y=y x \cdot e
$$

These and other basic definitions are collected in the Table 1.

\section{Isotopy}

If $\cdot$ and $\times$ are quasigroups (on $S$, respectively $T$ ) and $f, g, h: S \rightarrow T$ bijections such that $f(x y)=g x \times h y$ then we say that $\cdot$ and $\times$ are isotopic and that $(f, g, h)$ is an isotopy. Isotopy is a generalization of isomorphism. Isotopic image of a quasigroup is again a quasigroup. A loop isotopic to a group is isomorphic to it.

Every quasigroup is isotopic to some loop i.e., it is a loop isotope.

Definition 2.1. A quasigroup · on $S$ is a group (Abelian group, Boolean group) isotope iff $x y=f x+g y$, where + is a group (Abelian group, Boolean group) on $S$, while $f$ and $g$ are permutations. tity:

Belousov [1] characterized quasigroups which are group isotopes, by the iden-

$$
x(y \backslash(z / u) v)=(x(y \backslash z) / u) v
$$

and the quasigroups which are Abelian group isotopes, by the identity:

$$
x \backslash y(u \backslash v)=u \backslash y(x \backslash v) .
$$

Falconer $[8]$ proved that quasigroups are Boolean group isotopes if they satisfy the equation:

$$
x y / z=x z / y .
$$


TABLE 1

\begin{tabular}{|c|c|c|c|}
\hline variety & notation & defining identities & name \\
\hline Quasigroups & $Q$ & $x=x$ & $(Q)$ \\
\hline Loops & $\Lambda$ & $x \backslash x=y / y$ & $(u)$ \\
\hline Pointed loops & $\Lambda_{1}$ & $e x=x e=x$ & $(1)$ \\
\hline $\begin{array}{c}\text { Commutative } \\
\text { quasigroups }\end{array}$ & $C$ & $x y=y x$ & $(C)$ \\
\hline $\begin{array}{c}\text { Unipotent } \\
\text { quasigroups }\end{array}$ & $U$ & $x x=y y$ & $(U)$ \\
\hline $\begin{array}{c}\text { Unipotent pointed } \\
\text { quasigroups }\end{array}$ & $U_{1}$ & $x x=e$ & $\left(U_{1}\right)$ \\
\hline $\begin{array}{c}\text { Unipotent commutative } \\
\text { quasigroups }\end{array}$ & $C U$ & $(C),(U)$ & - \\
\hline $\begin{array}{c}\text { Skew symmetric } \\
\text { quasigroups }\end{array}$ & $U 1$ & $x y \cdot y x=e$ & $(U 1)$ \\
\hline$b 0$-quasigroups & $b 0$ & $e x=x e$ & $(b 0)$ \\
\hline$b 1$-quasigroups & $b 1$ & $e \cdot x y=y x \cdot e$ & $(b 1)$ \\
\hline $\begin{array}{c}\text { Unipotent } \\
b 0 \text {-quasigroups }\end{array}$ & $U b 0$ & $(U),(b 0)$ & - \\
\hline $\begin{array}{c}\text { Unipotent } \\
b 1 \text {-quasigroups }\end{array}$ & $U b 1$ & $(U),(b 1)$ & - \\
\hline$T S$-quasigroups & $T S$ & $x y=x \backslash y=x / y$ & $(T S)$ \\
\hline$T S$-loops & $T S \Lambda$ & $(T S),(\Lambda)$ & - \\
\hline Groups & $G$ & $x \cdot y z=x y \cdot z$ & $(G)$ \\
\hline Abelian groups & $A$ & $(G),(C)$ & - \\
\hline Boolean groups & $B$ & $(G),(U)$ & - \\
\hline
\end{tabular}

Definition 2.2. A quasigroup · on $S$ is left (right) linear over a group (Abelian group, Boolean group) iff $x y=A x+f y(x y=f x+A y)$, where + is a group (Abelian group, Boolean group) on $S, A$ is an automorphism of + and $f$ is a permutation.

Belyavskaya and Tabarov proved in [4] that a quasigroup is left (right) linear over a group iff it satisfies the identity

$$
x(u \backslash y) \cdot z=x(u \backslash u) \cdot(u \backslash y z)
$$

respectively the identity

$$
x \cdot(y / u) z=(x y / u) \cdot(u / u) z .
$$

Of particular interest are quasigroups which are left (right) linear over groups as well as unipotent. They have the form: $x y=A x-A y+c(x y=c-A x+A y)$ where $c \in S$.

Here, as usual, $x-y$ stands for $x+(-y)$, where $-y$ is the inverse element of $y$. 
If a quasigroup is left (right) linear over an Abelian group it is sometimes called an LT-quasigroup (RT-quasigroup).

Definition 2.3. A quasigroup - on $S$ is linear over a group (Abelian group, Boolean group) iff $x y=A x+c+B y$, where + is a group (Abelian group, Boolean group) on $S, A$ and $B$ are automorphisms of + and $c$ is a constant.

Note that in unipotent quasigroups being left (right) linear over an Abelian group is the same as being linear over the same group.

Quasigroups which are linear over a group are characterized in Belyavskaya, Tabarov [5]. Namely, they proved that a quasigroup - is linear over a group iff it satisfies the identity:

$$
x y \cdot u v=x u \cdot\left(\alpha_{u} y \cdot v\right)
$$

where $\alpha_{u} y=[u \backslash((u / u) y \cdot u] /(u \backslash u)$.

Quasigroup linear over an Abelian group is also called a T-quasigroup.

\section{Functional equations on quasigroups}

We emphasize that we shall consider only quasigroup equations.

Definition 3.1. Functional equation $s=t$ is quadratic if every variable appears exactly twice in $s=t$. Quadratic equation is balanced (or linear) if every variable appears exactly once in $s$ and once in $t$.

EXAMPLE 3.1. The following are various functional equations.

$$
\begin{aligned}
x y \cdot z & =x \cdot y z & & \text { (associativity) } \\
x y \cdot z u & =x z \cdot y u & & \text { (mediality) } \\
x y \cdot z u & =(x z \cdot y) u & & \text { (pseudomediality) } \\
x \cdot y z & =x y \cdot x z & & \text { (left distributivity) } \\
x y \cdot y z & =x z & & \text { (transitivity) }
\end{aligned}
$$

Associativity, mediality and pseudomediality are balanced, transitivity is quadratic but not balanced and left distributivity is not even quadratic.

We briefly mention a few attempts to solve various classes of equations and give better insight into their mutual relationships.

In the paper [10] Ježek and Kepka solved all balanced linear equations with up to three variables. The result was that these 27 equations define (by itself or combined) exactly 11 quasigroup varieties. Duplak in [7] generalized this result by allowing both division operations in equations (with up to three variables) and obtained exactly 55 varieties as solutions.

Belousov defined in [3] an important class of balanced equations which were named Belousov equations by Krapež and Taylor in [13]. A balanced equation $s=t$ is Belousov if for every subterm $p$ of $s(t)$ there is a subterm $q$ of $t(s)$ such 
that $p$ and $q$ have exactly the same variables. Examples of Belousov equations are:

(Q)

$$
\begin{aligned}
x & =x \\
x y & =x y \\
x y & =y x \\
x \cdot y z & =z y \cdot x \\
x y \cdot u v & =v u \cdot y x \\
x y \cdot(z u \cdot v w) & =(u z \cdot w v) \cdot y x
\end{aligned}
$$

The equation $(\mathrm{Q})$ and all equations $t=t$ are trivial. Belousov equations not equivalent to (Q) are nontrivial. A quasigroup satisfying a set of Belousov equations, not all of them trivial, is a Belousov quasigroup.

The characteristic property of Belousov equations is:

TheOrem 3.1 (Krapež [12]). A balanced quasigroup equation $s=t$ is Belousov:

- iff $s=t$ is a consequence of the theory of commutative quasigroups

- iff there is an equation $E q(\cdot, *)$ which is true in all quasigroups and $s=t$ is $E q(\cdot, \cdot)$.

The operation $*$ is the so called dual operation of $\cdot$ and is defined by $x * y=y \cdot x$. The symbol $*$ is considered not to belong to the language of quasigroups.

The importance of Belousov equations stems from the following:

TheOREM 3.2 (Krapež [12], Belousov [3]). A quasigroup satisfying a balanced but not Belousov equation is isotopic to a group.

Belousov equations are solved in [14] using polynomials from $\mathbb{Z}_{2}[x]$.

Krapež and Taylor defined gemini equations in [15].

Definition 3.2. A quadratic equation $s=t$ is gemini if it is a consequence of the theory of TS-loops.

The following theorem generalizes the Theorem 3.2:

TheOREM 3.3 (Krapež, Taylor [15]). A quasigroup satisfying a quadratic but not gemini equation is isotopic to a group.

\section{Quadratic level equations}

Equations under consideration are quadratic and of the form:

$$
x_{1} x_{2} \cdot x_{3} x_{4}=x_{5} x_{6} \cdot x_{7} x_{8}
$$

where $x_{i} \in\{x, y, u, v\}(1 \leqslant i \leqslant 8)$. All variables are on the same 'level' above roots of the left-(right-)hand side trees of terms in (L2), so we call equations (L2) level equations.

We note that, although quasigroups might be defined equationally, using multiplication $(\cdot)$ and both division operations $(\backslash$ and $/$ ), the equations which we consider contain the multiplication symbol only.

There are 105 such equations. We list them all. 


\begin{tabular}{|c|c|c|c|c|c|}
\hline (4.1) & $x x \cdot y y=u u \cdot v v$ & $(4.3$ & $x y \cdot y u=x v \cdot v u$ & $(4.71)$ & $x y \cdot u$ \\
\hline$(4.2)$ & $x x \cdot y y=u v \cdot u v$ & $(4.37)$ & $x y \cdot y u=u x \cdot v v$ & $(4.72)$ & $x y \cdot u u=x v \cdot v y$ \\
\hline .3$)$ & $x x \cdot y y=u v \cdot v u$ & $(4.38)$ & $x y \cdot y u=u v \cdot x v$ & 4.73) & $x y \cdot u u=y x \cdot v v$ \\
\hline .4$)$ & $x x \cdot y u=y u \cdot v v$ & $(4.3$ & $x y \cdot y u=u v \cdot v x$ & 4.74) & $x y \cdot u u=y v \cdot x v$ \\
\hline & $x x \cdot y u=y v \cdot u v$ & ( & $x y \cdot y u=v x \cdot u v$ & & $x y \cdot u u=y v \cdot v x$ \\
\hline & $x x \cdot y u=y v \cdot v u$ & & $x y \cdot y u=v x \cdot v u$ & & $x \cdot y v$ \\
\hline & $x x \cdot y u=u y \cdot v v$ & & $x y \cdot y u=v u \cdot x v$ & & $x \cdot v y$ \\
\hline & $x x \cdot y u=u v \cdot y v$ & & $x y \cdot y u=v u \cdot v x$ & & $x y \cdot u u=v y \cdot x v$ \\
\hline 9) & $x x \cdot y u=u v \cdot v y$ & ( & $x y \cdot y u=v v \cdot x u$ & & $x y \cdot u u=v y \cdot v x$ \\
\hline$(4.10)$ & $x x \cdot y u=v y \cdot u v$ & $(4.45)$ & $x y \cdot y u=v v \cdot u x$ & $(4.80)$ & $x y \cdot u u=v v \cdot x y$ \\
\hline$(4.11)$ & $x x \cdot y u=v y \cdot v u$ & $(4.46)$ & $x y \cdot u x=y u \cdot v v$ & $(4.81)$ & $x y \cdot u u=v v \cdot y x$ \\
\hline$(4.12)$ & $x x \cdot y u=v u \cdot y v$ & $(4.47)$ & $x y \cdot u x=y v \cdot u v$ & $(4.82)$ & $x y \cdot u v=x y \cdot u v$ \\
\hline$(4.13)$ & $x x \cdot y u=v u \cdot v y$ & $(4.48)$ & $x y \cdot u x=y v \cdot v u$ & $(4.83)$ & $x y \cdot u v=x y \cdot v u$ \\
\hline$(4.14)$ & $x x \cdot y u=v v \cdot y u$ & $(4.49)$ & $x y \cdot u x=u y \cdot v v$ & $(4.84)$ & $x y \cdot u v=x u \cdot y v$ \\
\hline$(4.15)$ & $x x \cdot y u=v v \cdot u y$ & $(4.50)$ & $x y \cdot u x=u v \cdot y v$ & $(4.85)$ & $x y \cdot u v=x u \cdot v y$ \\
\hline$(4.16)$ & $x y \cdot x y=u u \cdot v v$ & $(4.51)$ & $x y \cdot u x=u v \cdot v y$ & $(4.86)$ & $x y \cdot u v=x v \cdot y u$ \\
\hline .17$)$ & $x y \cdot x y=u v \cdot u v$ & $(4.52)$ & $x y \cdot u x=v y \cdot u v$ & $(4.87)$ & $x y \cdot u v=x v \cdot u y$ \\
\hline$(4.18)$ & $x y \cdot x y=u v \cdot v u$ & $(4.53)$ & $x y \cdot u x=v y \cdot v u$ & $(4.88)$ & $x y \cdot u v=y x \cdot u v$ \\
\hline$(4.19)$ & $x y \cdot x u=y u \cdot v v$ & $(4.54)$ & $x y \cdot u x=v u \cdot y v$ & $(4.89)$ & $x y \cdot u v=y x \cdot v u$ \\
\hline$(4.20)$ & $x y \cdot x u=y v \cdot u v$ & $(4.55)$ & $x y \cdot u x=v u \cdot v y$ & & $x y \cdot u v=y u \cdot x v$ \\
\hline$(4.21)$ & $x y \cdot x u=y v \cdot v u$ & & $x y \cdot u x=v v \cdot y u$ & & $x y \cdot u v=y u \cdot v x$ \\
\hline$(4.22)$ & & $(4.57)$ & & $(4.92)$ & $x y \cdot u v=y v \cdot x u$ \\
\hline$(4.23)$ & $x y \cdot x u=u v \cdot y v$ & $(4.58)$ & $x y \cdot u y=x u \cdot v v$ & & \\
\hline$(4.24)$ & $x y \cdot x u=u v \cdot v y$ & $(4.59)$ & $x y \cdot u y=x v \cdot u v$ & $(4.94)$ & $x y \cdot u v=u x \cdot y v$ \\
\hline$(4.25)$ & $x y \cdot x u=v y \cdot u v$ & $(4.60)$ & $x y \cdot u y=x v \cdot v u$ & $(4.95)$ & $x y \cdot u v=?$ \\
\hline$(4.26)$ & $x y \cdot x u=v y \cdot v u$ & $(4.61)$ & $x y \cdot u y=u x \cdot v v$ & $(4.96)$ & $x y \cdot u v=u y \cdot x v$ \\
\hline$(4.27)$ & $x y \cdot x u=v u \cdot y v$ & $(4.62)$ & $x y \cdot u y=u v \cdot x v$ & $(4.97)$ & $x y \cdot u v=u y \cdot v x$ \\
\hline$(4.28)$ & $x y \cdot x u=v u \cdot v y$ & $(4.63)$ & $x y \cdot u y=u v \cdot v x$ & $(4.98)$ & $x y \cdot u v=u v \cdot x y$ \\
\hline$(4.29)$ & $x y \cdot x u=v v \cdot y u$ & $(4.64)$ & $x y \cdot u y=v x \cdot u v$ & $(4.99)$ & $x y \cdot u v=u v \cdot y x$ \\
\hline$(4.30)$ & $x y \cdot x u=v v \cdot u y$ & $(4.65)$ & $x y \cdot u y=v x \cdot v u$ & $(4.100)$ & $x y \cdot u v=v x \cdot y u$ \\
\hline$(4.31)$ & $x y \cdot y x=u u \cdot v v$ & $(4.66)$ & $x y \cdot u y=v u \cdot x v$ & $(4.101)$ & $x y \cdot u v=v x \cdot u y$ \\
\hline$(4.32)$ & $x y \cdot y x=u v \cdot u v$ & $(4.67)$ & $x y \cdot u y=v u \cdot v x$ & $(4.102)$ & $x y \cdot u v=v y \cdot x u$ \\
\hline$(4.33)$ & $x y \cdot y x=u v \cdot v u$ & $(4.68)$ & $x y \cdot u y=v v \cdot x u$ & $(4.103)$ & $x y \cdot u v=v y \cdot u x$ \\
\hline$(4.34)$ & $x y \cdot y u=x u \cdot v v$ & $(4.69)$ & $x y \cdot u y=v v \cdot u x$ & $(4.104)$ & $x y \cdot u v=v u \cdot x y$ \\
\hline$(4.35)$ & $x y \cdot y u=x v \cdot u v$ & $(4.70)$ & $x y \cdot u u=x y \cdot v v$ & $(4.105)$ & $x y \cdot u v=v u \cdot y x$ \\
\hline
\end{tabular}


The last 24 equations are balanced. Their solutions are given in [9] and [21].

In this paper we give solutions of the remaining 81 equations. This, among other things, gives a contribution to the knowledge of a part of the lattice of quasigroup varieties.

\section{Balanced equations}

For the balanced equations (4.82)-(4.105) we have ([9], [21]):

- The equation (4.82) is trivial i.e., all quasigroups are solutions.

- The equations (4.83), (4.88), (4.89), (4.98), (4.99) and (4.104) are all equivalent to $(\mathrm{C})$; solutions are commutative quasigroups.

- The equation (4.105) is equivalent to (B11); solutions belong to the Belousov variety $B 11$.

- The equation (4.84) defines medial quasigroups constituting the variety $M$ of $T$-quasigroups with $A B=B A$ (see $[\mathbf{2 2}]$ and $[\mathbf{1 8}]$ ).

- The equation (4.103) defines paramedial quasigroups constituting the variety $P$ of $T$-quasigroups with $A A=B B$ (see [19] and [11]).

- The equations (4.85)-(4.87), (4.90)-(4.97) and (4.100)-(4.102) are equivalent to commutative (para)mediality; solutions constitute the variety $T 1$ of commutative $T$-quasigroups (i.e., with $A=B$ ).

Equations (4.84) and (4.103) together define the new variety $T 11$. The variety $T 11$ can be also defined by a single (balanced) equation, for example $(x y \cdot u v)(p q \cdot r s)$ $=(x u \cdot y v)(s q \cdot r p)$, but this equation has eight variables.

Every other subset of equations (4.82)-(4.105) gives a variety equal to the one given above. The mutual relationship between these varieties is given by the following graph:

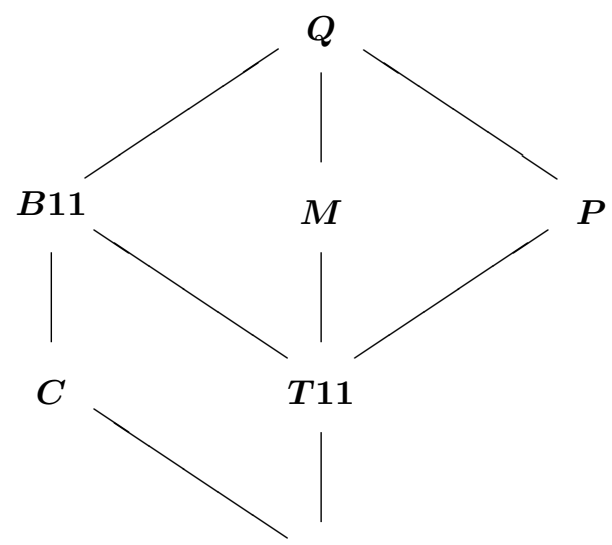

$T 1$

Figure 1. Varieties of quasigroups defined by balanced equations with four variables 


\section{Gemini equations}

Equations which are both gemini and balanced are Belousov. We saw that there are eight such equations which separate into three classes of mutually nonequivalent equations: $(\mathrm{Q}),(\mathrm{C})$ and $(\mathrm{B} 11)$.

It is easy to check that there are 17 gemini equations which are not balanced. They are: (4.1)-(4.4), (4.7), (4.14)-(4.18), (4.31)-(4.33), (4.70), (4.73), (4.80) and (4.81).

Lemma 6.1. Let $s=t$ be a quadratic level equation with four variables and let the square of a variable be a subterm of $s$ or $t$. A quasigroup satisfying $s=t$ must be unipotent.

Proof. Let $s=t$ be $x x \cdot v_{1} v_{2}=v_{3} v_{4} \cdot v_{5} v_{6}$ where $v_{i} \in\{y, u, v\}(i=1, \ldots, 6)$. Replace $y, u$ and $v$ by $a \in S$ and $a a$ by $e$. Then $x x \cdot e=e e$ i.e., $x x=e$.

Other cases follow by duality and/or symmetry of $=$.

LEMma 6.2. Let $s=t$ be a quadratic level equation with four variables and let $s(t)$ be the square of the product of two variables. A quasigroup satisfying $s=t$ must be unipotent.

Proof. Let $s=x y \cdot x y$. Replace $x y$ by $z$ and $u, v$ by $a$. Let $e=a a \cdot a a$. Then $z z=e$. The other case $(t=u v \cdot u v)$ is similar.

According to Lemma 6.1 and Lemma $6.2,16$ out of 17 gemini equations imply unipotency. The only exception is (4.33) which is independent of unipotency (for the proof see $[\mathbf{1 6}])$.

Theorem 6.1. Equations (4.1), (4.2), (4.14), (4.16), (4.17) and (4.70) are all equivalent to unipotency.

Proof. All equations imply unipotence. The converse is easy.

Lemma 6.3. An unipotent (pointed) quasigroup is skew symmetric iff it is commutative.

TheOREM 6.2. Equations (4.3), (4.15), (4.18), (4.31), (4.32) and (4.73) are all equivalent to commutative unipotency.

Proof. The unipotency follows. Replacing squares by $e$ and simplifying, we get either commutativity or skew symmetry. The converse is trivial.

The proofs of the theorems 6.3-6.5 are all straightforward.

TheOrem 6.3. Equations (4.4) and (4.80) are equivalent to (Ub0) as well as to the equation $x x \cdot y=y \cdot z z$.

TheOrem 6.4. Equations (4.7) and (4.81) are equivalent to (Ub1).

Theorem 6.5. Equation (4.33) is equivalent to (U1). 


\section{Group isotopes}

The 25 equations from the preceding section are gemini. The remaining 80 equations are not gemini and by the Theorem 3.3 have solutions which are group isotopes. In this section we give general solutions for ten of them.

TheOREM 7.1. Equations (4.8), (4.58), (4.59), (4.69) and (4.71) are mutually equivalent with all solutions unipotent quasigroups left linear over groups. More precisely, their general solution is given by:

$$
x y=A x-A y+e
$$

where $+i$ is an arbitrary group (on $S$ ), $A$ is an automorphism of + and $e$ is any element of $S$.

Proof. The quasigroup - defined by: $x y=A x-A y+e$ where + is a group, $A$ an automorphism of + and $e \in S$, is a solution of (4.8).

Conversely, assume that a quasigroup - on $S$ satisfies (4.8). Then there is a group + and permutations $f, g$ such that $x y=f x+g y$. Let $a \in S$ and $a a=e$. Put $y=u=v=a$. Then $x x=e$ and $f x+g x=e$ i.e., $g x=-f x+e$. Therefore $x y=f x-f y+e$. Substituting in (4.8) and reducing, we get $f(y-u+e)=$ $f(x+y)-f(x+u)+f e$. For $x=0$ we have

$$
f(y-u+e)=f y-f u+f e .
$$

Let $u=0$ and $b=f 0$. Then $f(y+e)=f y-b+f e$ and (7.1) yields $f(y-u)-b=$ $f y-f u$. It follows that $f(-u)=b-f u+b$ and $f(y+z)=f y-b+f z$. But then $A x=f x-b$ is an automorphism of + and $x y=A x-A y+e$ as required.

The proof for the other four equations is similar.

Dually:

ThEOREM 7.2. Equations (4.11), (4.22), (4.26), (4.29) and (4.79) are mutually equivalent with all solutions unipotent quasigroups right linear over groups. More precisely, their general solution is given by:

$$
x y=e-A x+A y
$$

where + is an arbitrary group (on $S$ ), $A$ is any automorphism of + and $e$ is any element of $S$.

In the next section we prove that the remaining 70 equations have solutions which must be Abelian group isotopes.

\section{Abelian group isotopes}

In the section 5 we saw that there are 16 equations which are balanced and nonBelousov. They separate into three classes of mutually non-equivalent equations: $(\mathrm{M}),(\mathrm{P})$ and $(\mathrm{T} 1)$. All solutions are Abelian group isotopes, moreover they are $T$-quasigroups.

For the non-balanced case we need the following criterion of Krstić [17]. 
THEOREM 8.1. Let $E$ be a quadratic quasigroup equation with a solution which must be a group isotope. It is an Abelian group isotope iff $E^{\alpha \beta}$ is nontrivial group equation for all $\alpha, \beta \in\{+1,-1\}$.

The equation $E^{\alpha \beta}$ is the group equation obtained from $E$ by replacing every subterm $s \cdot t$ of $E$ by $s^{\alpha} \cdot t^{\beta}$.

In case of quadratic level equations (L2), we have:
$\left((L 2)^{+1+1}\right)$
$x_{1} x_{2} x_{3} x_{4}=x_{5} x_{6} x_{7} x_{8}$
$\left((L 2)^{+1-1}\right)$
$x_{1} x_{2}^{-1} x_{4} x_{3}^{-1}=x_{5} x_{6}^{-1} x_{8} x_{7}^{-1}$
$\left((L 2)^{-1+1}\right)$
$x_{2}^{-1} x_{1} x_{3}^{-1} x_{4}=x_{6}^{-1} x_{5} x_{7}^{-1} x_{8}$
$\left((L 2)^{-1-1}\right)$
$x_{2} x_{1} x_{4} x_{3}=x_{6} x_{5} x_{8} x_{7}$

Therefore:

Corollary 8.1. Let a solution of $(L 2)$ be a group isotope. It is an Abelian group isotope iff $\left((L 2)^{+1+1}\right),\left((L 2)^{+1-1}\right),\left((L 2)^{-1+1}\right),\left((L 2)^{-1-1}\right)$ are all nontrivial group equations.

TheOREm 8.2. All solutions of the equations (4.5), (4.6), (4.9), (4.10), (4.12), (4.13), (4.19)-(4.21), (4.23)-(4.25), (4.27), (4.28), (4.30), (4.34)-(5.57), (4.60)(4.68), (4.72), (4.74)-(4.78), (4.84)-(4.87), (4.90)-(4.97), (4.100)-(4.103) are Abelian group isotopes. Moreover, they are T-quasigroups.

Proof. (a) The first part of the proof consists of applying Corollary 8.1 and checking four group equations for every quasigroup equation - seventy times. We do it for just one equation: (4.23).
$\left((4.23)^{+1+1}\right)$
$x y x u=u v y v$
$\left((4.23)^{+1-1}\right)$
$x y^{-1} u x^{-1}=u v^{-1} v y^{m 1}$
$\left((4.23)^{-1+1}\right)$
$y^{-1} x x^{-1} u=v^{-1} u y^{-1} v$
$\left((4.23)^{-1-1}\right)$
$y x u x=v u v y$

Neither of the above equations is trivial in groups and therefore, by the Corollary 8.1, the solution of (4.23) must be an Abelian group isotope.

(b) Since every equation is non-gemini there is a pair of variables $x, y$ such that the term $x y$ appears in the equation as a subterm just once, while $y x$ does not appear as a subterm at all. Replacing other variables by elements from $S$, we conclude that there are permutations $P$ and $Q$ on $S$ such that either $f(x+y)=$ $P x+Q y$ or $g(x+y)=P x+Q y$. Assume

$$
f(x+y)=P x+Q y
$$

For $x=0$ (where 0 is the unit of the Abelian group + ) we get $f y=P 0+Q y$ i.e., $Q y=f y-P 0$ and similarly $P x=f x-Q 0$. If we put this back in the equation 
(8.1) we get $f(x+y)=f x+f y-(P 0+Q 0)$ i.e., $A x=f x-(P 0+Q 0)$ is an automorphism of + .

Analogously, there is a $b \in S$ such that $B x=g x+b$ is an automorphism of + so $x y=f x+g y=A x+B y+(P 0+Q 0-b)$ i.e., · is a $T$-quasigroup.

LEMMA 8.1. If $x y=A x+B y+c$, where + is an Abelian group, $A$ and $B$ its automorphisms and $c$ an arbitrary element, the equation (L2) is equivalent to

$$
A A x_{1}+A B x_{2}+B A x_{3}+B B x_{4}=A A x_{5}+A B x_{6}+B A x_{7}+B B x_{8}
$$

Proof. Just replace every product $x y$ in (L2) by $A x+B y+c$ and subtract $A c+B c+c$ from both sides.

We give general solutions for six equations which are not balanced.

THEOREM 8.3. Equations (4.23) and (4.67) are mutually equivalent and have as a general solution unipotent quasigroups linear over Abelian groups i.e.,

$$
x y=A x-A y+c
$$

where + is an arbitrary Abelian group (on $S$ ), $A$ is any automorphism of + and $c$ is any element of $S$.

Proof. According to Lemma 8.1, equation (4.23) is equivalent to

$$
\begin{aligned}
& A A x+A B y+B A x+B B u=A A u+A B v+B A y+B B v, \quad \text { i.e., } \\
& (A A+B A) x+(A B-B A) y+(B B-A A) u-(A B+B B) v=0 .
\end{aligned}
$$

If we define $O(x)=0$ for all $x \in S$ we see that the above equation is equivalent to the system:

$$
\left\{\begin{array}{l}
A A+B A=O, \\
A B-B A=O, \\
B B-A A=O \\
A B+B B=O
\end{array}\right.
$$

of operator identities. Since $A$ and $B$ are automorphisms, the first equality is equivalent to $A+B=O$ (i.e., $B(x)=-A(x))$. Checking, we see that the whole system is equivalent to $A+B=O$ as needed.

Similarly, equation (4.67) reduces to

$$
\begin{aligned}
& (A A-B B) x+(A B+B B) y+(B A-A B) u-(A A+B A) v=0 \text { i.e., } \\
& \left\{\begin{array}{l}
A A-B B=O, \\
A B+B B=O, \\
B A-A B=O, \\
A A+B A=O
\end{array}\right.
\end{aligned}
$$

which is equivalent to $A+B=O$ as well. 
THEOREM 8.4. All solutions of the equation (4.36) are quasigroups linear over Abelian groups. More precisely, their general solution is given by:

$$
x y=A x+B y+c
$$

where + is an arbitrary Abelian group (on $S$ ), $A$ and $B$ are automorphisms of + , such that $A B+B A=O$, and $c$ is any element of $S$.

Proof. The equation (4.36) reduces to:

$$
\left\{\begin{array}{l}
A A-A A=O, \\
A B+B A=O, \\
B B-B B=O, \\
A B+B A=O
\end{array}\right.
$$

which is equivalent to $A B+B A=O$.

Quasigroups which satisfy the equation (4.36) will be called intermedial.

THEOREM 8.5. All solutions of the equation (4.52) are quasigroups linear over Abelian groups. More precisely, their general solution is given by:

$$
x y=A x+B y+c
$$

where + is an arbitrary Abelian group (on $S$ ), $A$ and $B$ are automorphisms of + , such that $A A+B B=O$, and $c$ is any element of $S$.

Proof. The equation (4.52) reduces to:

$$
\left\{\begin{array}{l}
A A+B B=O, \\
A B-A B=O, \\
B A-B A=O, \\
A A+B B=O
\end{array}\right.
$$

which is equivalent to $A A+B B=O$.

Quasigroups which satisfy the equation (4.52) will be called extramedial.

THEOREM 8.6. The equation (4.39) has a general solution:

$$
x y=A x+B y+c
$$

where + is an arbitrary Abelian group (on $S$ ), $A$ and $B$ are automorphisms of + , such that $A A=B B$ and $A B+B A=O$, and $c$ is any element of $S$.

Proof. The equation (4.39) is equivalent to:

$$
\left\{\begin{array}{l}
A A-B B=O, \\
A B+B A=O, \\
B B-A A=O, \\
A B+B A=O
\end{array}\right.
$$

which reduces to

$$
\left\{\begin{array}{c}
A A=B B, \\
A B+B A=O .
\end{array}\right.
$$


THEOREM 8.7. The equation (4.54) has a general solution:

$$
x y=A x+B y+c
$$

where $+i$ s an arbitrary Abelian group (on $S$ ), $A$ and $B$ are automorphisms of + , such that $A A+B B=O$ and $A B=B A$, and $c$ is any element of $S$.

Proof. The equation (4.54) is equivalent to:

$$
\left\{\begin{array}{l}
A A+B B=O, \\
A B-B A=O, \\
B A-A B=O, \\
A A+B B=O
\end{array}\right.
$$

which reduces to

$$
\left\{\begin{array}{c}
A A+B B=O \\
A B=B A
\end{array}\right.
$$

\section{Boolean group isotopes}

In this section we prove that the remaining 48 equations have solutions which must be Boolean group isotopes.

Theorem 9.1. Equations (4.5), (4.6), (4.9), (4.10), (4.12), (4.13), (4.19)(4.21), (4.24), (4.25), (4.27), (4.28), (4.30), (4.34), (4.35), (4.37), (4.38), (4.40)(4.51), (4.53), (4.55)-(4.57), (4.60)-(4.66), (4.68), (4.72), (4.74)-(4.78) are all mutually equivalent. Their general solution is given by:

$$
x y=A x+A y+e
$$

where $+i s$ an arbitrary Boolean group (on $S), A$ is an automorphism of + and $e$ is any element of $S$.

Proof. We give the proof for the equation:

$$
x y \cdot u y=x v \cdot v u .
$$

The proofs for other equations are just minor variants of the given one.

By the Theorem 8.2 and Corrolary 8.1, the equation (4.60) is equivalent to:

$$
\left\{\begin{array}{l}
A A-A A=O, \\
A B+B B=O, \\
B A-B B=O, \\
A B+B A=O
\end{array}\right.
$$

which reduces to

$$
\left\{\begin{array}{c}
A=B \\
A+B=O .
\end{array}\right.
$$

The last system is equivalent to $x+x=0$. 


\section{Conclusions}

We saw that 105 quadratic level identities (equations) with four variables define 19 varieties: $Q, U 1, B 11, C, U, U b 0, U b 1, C U, L L U, R L U, M, P, E, I, M E, P I$, $T 1, D 1, B T 1$. In this paper we gave general solutions of all 105 quadratic level equations. We proved:

- Every quadratic level equation is equivalent to the one of 19 quasigroup identities which define 19 quasigroup varieties.

- There are 25 gemini equations. Eight are balanced and therefore Belousov defining three varieties $(Q, B 11$ and $C)$. Seventeen are non-balanced and they define five varieties $(U, U b 0, U b 1, C U$ and $U 1)$.

- There are 80 equations which force quasigroups satisfying them to be group isotopes. But only ten of them do not force this group to be Abelian. They define two varieties $L L U$ and $R L U$.

- There are 70 equations which force quasigroups satisfying them to be Abelian group isotopes. But only 22 equations do not force this group to be Boolean. The 16 balanced of these 22 equations define varieties $M, P$ and $T 1$. The remaining six equations define varieties $D 1, E, I, P I$ and $M E$.

- The variety BT1 of Boolean group isotopes is defined by the each of the remaining 48 equations.

In the sequel $[\mathbf{1 6}]$ of this paper, we shall prove:

- That the above 19 varieties are actually distinct one from the other.

- That there are seven (mutually nonequivalent) systems of two equations each, which are not equivalent to any single equation with four variables. However, these seven systems are each equivalent to a single quadratic level equation with eight variables.

- That the conjunction of any subset of 105 equations gives one of the above 26 varieties.

- That the ordening 'being a subset' on the set $Q_{4}{ }^{1}$ of the above varieties is a lattice ordering. However, this lattice is not a sublattice of the lattice of all varieties of quasigroups.

The diagram of the lattice $Q_{4}$ will also be given.

\section{References}

[1] V.D. Belousov, Balanced identities on Quasigroups (Russian), Mat. Sb. 70 (112) (1966).

[2] V.D. Belousov, Foundations of the Theory of Quasigroups and Loops (Russian), Nauka, Moscow, 1967; MR 36\#1569, Zbl 163.01801.

[3] V.D. Belousov, Quasigroups with completely reducible balanced identities (Russian), Mat. Issled. 83 (1985), 11-25.

[4] G. B. Belyavskaya, A.H. Tabarov, Nuclei and the centre of linear quasigroups (Russian), Izvestija AN RM Matematika 3/6 (1991), 37-42.

[5] G. B. Belyavskaya, A. H. Tabarov, A characterization of linear and alinear quasigroups (Russian), Diskr. Mat. 4/2 (1992), 142-147

\footnotetext{
${ }^{1}$ To avoid foundational issues, we work within a given universal set
} 
[6] O. Chein, H. O. Pflugfelder, J. D. H. Smith, Quasigroups and Loops: Theory and Applications, Sigma Series in Pure Math. 9, Heldermann, Berlin, 1990; MR 93g:21033, Zbl 0719.20036.

[7] J. Duplák, Quasigroups determined by balanced identities of length $\leqslant 6$, Czech. Math. J. 36(111) (1979).

[8] E. Falconer, Isotopy Invariants in Quasigroups, Trans. Amer. Math. Soc. 151/2 (1970), $511-526$.

[9] W. Förg-Rob, A. Krapež, Equations which preserve the height of variables, Aequat. Math. 70 (2005), 63-76.

[10] J. Ježek, T. Kepka, Varieties of quasigroups determined by short strictly balanced identities, Czech. Math. J. 29 (104) (1979).

[11] T. Kepka, P. Němec, T-quasigroups II, Acta Univ. Carol. Math. Phys. 12/2 (1971), 31-49.

[12] A. Krapež, On solving a system of balanced functional equations on quasigroups III, Publ. Inst. Math., Nouv. Sér. 26 (40) (1979), 145-156.

[13] A. Krapež, M. A. Taylor, Belousov equations on quasigroups, Aequat. Math. 34 (1987), 174185.

[14] A. Krapež, M. A. Taylor, Irreducible Belousov equations on quasigroups, Czech. Math. J. 43 (118) (1993), 157-175

[15] A. Krapež, M. A. Taylor, Gemini functional equations on quasigroups, Publ. Math. (Debrecen) 47/3-4 (1995), 283-292.

[16] A. Krapež, Quadratic level quasigroup equations with four variables II, in preparation.

[17] S. Krstić, Quadratic quasigroup identities, (Serbo-croatian), PhD thesis, University of Belgrade, 1985.

[18] D. C. Murdoch, Structure of abelian quasi-groups, Trans. Amer. Math. Soc. 49 (1941), 392409.

[19] P. Němec, T. Kepka, T-quasigroups I, Acta Univ. Carol. Math. Phys. 12/1 (1971), 39-49.

[20] H. O. Pflugfelder, Quasigroups and Loops: Introduction, Sigma Series in Pure Math. 8, Heldermann, Berlin, 1990; MR 93g:20132, Zbl 719.20036.

[21] M. Polonijo, On median-like identities, Quasigroups Relat. Syst. 13/2 (2005), 281-288.

[22] M. Toyoda, On axioms of linear functions, Proc. Imp. Acad. Jap. 17 (1941), 221-227.

Matematički institut SANU

Kneza Mihaila 36

11001 Beograd, p.p. 367

Serbia

sasa@mi.sanu.ac.yu 